\title{
Simultaneous Time-of-Flight Sensing and Photometric Stereo with a Single ToF Sensor
}

\author{
Changpeng Ti Ruigang Yang \\ Univ. of Kentucky \\ \{cti222, r.yang\}@uky.edu
}

\author{
James Davis \\ Univ. of California, Santa Cruz \\ davisecs.ucsc.edu
}

\author{
Zhigeng Pan \\ Hangzhou Normal Univ. \\ zgpan@hznu.edu.cn
}

\begin{abstract}
We present a novel system which incorporates photometric stereo with the Time-of-Flight depth sensor. Adding to the classic ToF, the system utilizes multiple point light sources that enable the capturing of a normal field whilst taking depth images. Two calibration methods are proposed to determine the light sources' positions given the ToF sensor's relatively low resolution. An iterative refinement algorithm is formulated to account for the extra phase delays caused by the positions of the light sources. We find in experiments that the system is comparable to the classic ToF in depth accuracy, and it is able to recover finer details that are lost due to the noise level of the ToF sensor.
\end{abstract}

\section{Introduction}

Full-frame time-of-flight (ToF) sensors have been introduced for several years. Developed for real-time acquisition of dynamic scenes, they typically have very fast shutter speed to take multiple shots of the scene rapidly. For example, ToF cameras based on phase-shift (such as PMD [1], SwissRanger [2]) take four snapshots to generate a metric depth map. In this paper, we develop novel techniques to allow Phase-shift-based ToF cameras to measure both metric distance and surface normal.

Our method starts with a simple addition of three more LED sources to the original camera. As shown in Figure 1, the LEDs are now placed away from the imaging sensors. Instead of illuminating the scene from the same light source four times to complete one acquisition cycle, we illuminate the scene with these four lights, one at a time. Based on four phase images from such a distributed light setup, new algorithms are developed to recover both the surface normal and the distance for each pixel.

The main contributions of this paper include: (1) a novel way to re-purpose the ToF sensor for both normal and depth capture, the required changes in hardware are low cost and small. (2) an algorithm to estimate time of flight from dis- tributed light sources, which provides an alternative solution to frequency modulation to use multiple ToF lights in the same environment; and (3) new methods to calibrate point light sources, which are specifically designed to lowresolution images such as these produced by today's ToF sensors.

We hope our method will inspire the next generation of ToF cameras that can produce significantly better depth maps than the current ones.

\section{Related Work}

There have been several works attempting to enhance range maps. In general these techniques are designed for general purpose depth enhancement and do not differentiate the underlying measuring principals. Only some of them [3] take into account the specific ToF sensor behavior. These techniques can mainly be categorized into two groups. The first group is based on image super-resolution techniques that utilize temporal redundancy, while the second group is mostly based on fusion with other devices to take advantage of spatial redundancy. Our approach falls into the second group. We will briefly discuss existing works in both categories.

Image super-resolution techniques haven been long studied [4]. With the increasing popularity of ToF sensors, these techniques have been adapted to accommodate the 2.5-D range images. Schuon et al [5] use a set of low resolution depth images that are captured from slightly displaced views and later registered. The enhancement task is formulated under a convex optimization framework with a data term to maintain the original measurement and a smoothness term that simultaneously preserves high frequency details and suppresses noises. Cui et al extend this technique by taking into account the specific characteristics of $\mathrm{ToF}$ sensors and incorporates a Nonlinear Anisotropic Regularizer to better preserve the local structures [3]. Both techniques take as input a set of depth maps captured without large view changes. Moreover, an image registration step, e.g. using optical flow [3], is required. 
Depth fusion schemes have been proposed to overcome the need of multiple frames captured temporally by incorporating extra devices to provide measurements captured simultaneously [6]. Moreover, different modalities are usually used to capture information that is not available in or complementary to the ToF range information. Instead of online temporal image registrations, usually only one offline calibration between sensors is need. Kopf et al [7] and Yang et al [6] extend the traditional Bilateral Filtering techniques [8] to incorporate the information from an extra color images corresponding to the depth map. Despite of the different modalities, impressive results have been achieved even very low resolution depth maps are used as input. The requirement of these approaches are usually high-resolution color images.

Fusion with Photometric Stereo has also been studied $[9,10]$. Nehab et al [9] use an iterative optimization scheme to combine position information from range maps and normal information from Photometric Stereo. It has been shown that the level of details can be greatly boosted via this fusion scheme. Zhang et al [10] further extend this technique to better preserve the depth continuities by using a Bilateral-like kernel in their Photometric Stereo formulation. Moreover, they show that this method can accommodate the degenerate regions with insufficient lights, such as the shadow regions. Despite of impressive results achieved by these methods, they require an extra setup dedicated for Photometric Stereo. Our approach builds upon this line of work. However, we greatly simplify the setup by only enhancing a regular ToF sensor with extra light sources without requiring any extra sensors or temporal information.

As will be illustrated in the next section, our hardware implementation of the depth fusion scheme utilizes distributed light sources for the ToF sensor. This requires the task of solving for depth given multiple light paths for each sensor pixel, which is related to analyzing multi-path interference. Among relevant literature, [11] and [12] take multiple measurements of the same scene under different modulation frequencies in order to separate reflectivity components, [13] achieves the reconstruction of multi-path interference by means of sparse regularization of the multiplemodulation frequency measurements. Also, [14] and [15] discuss obtaining transient images using the PMD camera, which inherently tackles the multi-path problem. It should be noted that our light path model differs from previous work, since we deal with multiple light sources instead of a single light source, and we also assume the Lambertian property for the scene, which allows us to focus only on the first light bounce.

\section{Time-of-Flight with Distributed Light Sources}

\subsection{Our System Overview}

As mentioned in Section 1, one of our major contributions is our novel ToF setup that is only slightly modified from a ToF sensor, but provides much more potentials for quality range sensing. Specifically, we take a regular ToF sensor and enhance it with four extra LED light sources, as shown in Fig. 1. Instead of taking intensity measurements from a single light sources. The intensity images are captured from the alternating distributed light sources. The extra distributed light sources enable us to perform Photometric Stereo to obtain a normal map, which reflects the surface details of the scene and will be later used to enhance the depth map. One could use the built-in light source with the sensor to acquire raw depth maps. However, it will result in more frames and therefore degrade the performance of high speed capturing. Instead, we use the data captured with the distributed lights for both Photometric Stereo reconstruction and ToF range sensing.

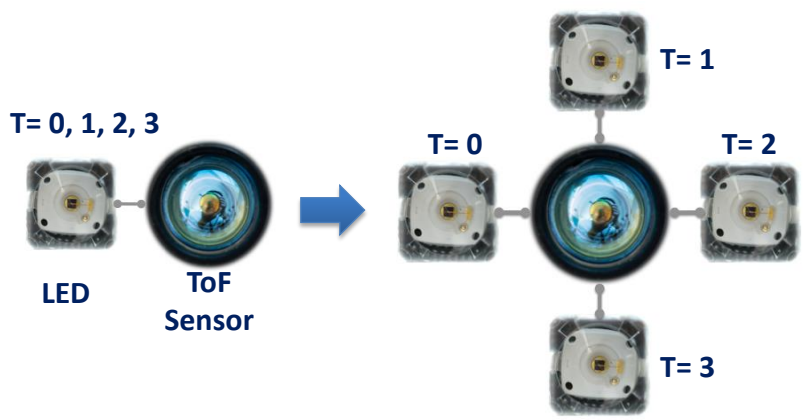

Figure 1. Illustration of our novel capturing setup that consists of a ToF sensor and four point light sources (right), comparing with the original ToF camera with one point light source (left).

Our algorithm consists of three main components: ToF sensing with distributed light sources, surface normal estimation via Photometric Stereo and finally a fusion scheme to combine these two sources of information. Fig. 2 shows the flowchart of our pipeline. In the rest of this section, we will first review the basic principle of ToF technique in Section 3.2 and then describe our method to accommodate the distributed light sources in our case in Section3.3. The fusion scheme will be discussed in Section 4. To enable our setup for close-range sensing, in this work, we model the light sources as point lights as opposed to directional lights in $[9,10]$. The strategies for light position calibration will be presented in Section 5 .

\subsection{Phase-Shift-based Time-of-Flight Principals}

A Photonic Mixing Device samples the correlation function $c(\tau)$ for a given phase shift $\tau$. 


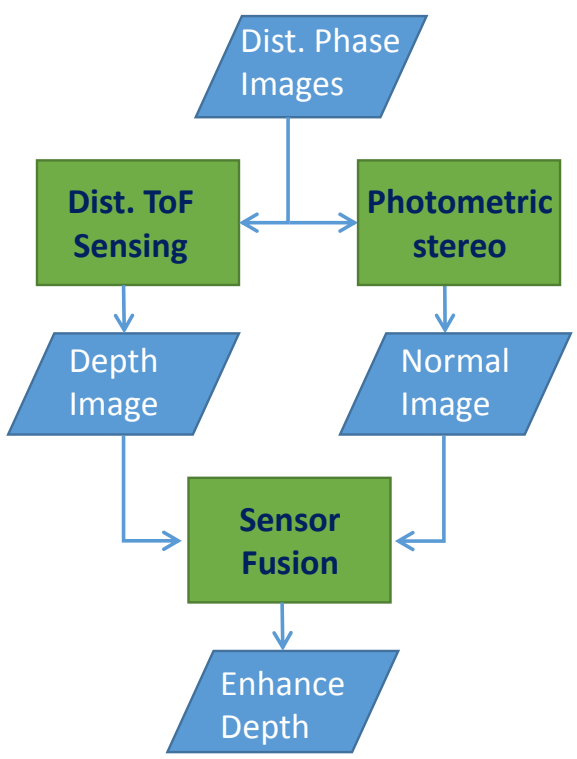

Figure 2. The overview of our depth enhancement framework.

$$
c(\tau)=\lim _{T->\infty} \int s(t) * g(t+\tau) d t
$$

For a sinusoidal signal, we can have the following by using some trigonometric calculus:

$s(t)=\cos (\omega t), g(t)=k+\alpha \cos (\omega t+\phi), c(t)=\alpha / 2 \cos (\omega t+\phi)$

where $\omega$ is the modulation frequency, $\alpha$ is the amplitude of the incident light and $\phi$ is the phase offset relating to the object distance. The modulation frequency uniquely defines the distance [1]. The demodulation of the correlation function is done by using several samples of $c(t)$ obtained by four sequential phase images $A_{i}=c(i \pi / 2)$ :

$\phi=\arctan \left(\frac{A_{3}-A_{1}}{A_{0}-A_{2}}\right), \alpha=\frac{\sqrt{\left(A_{3}-A_{1}\right)^{2}+\left(A_{0}-A_{2}\right)^{2}}}{2}$.

Our key insight in this approach is that the four phase images (e.g., $A_{0}, A_{1}, A_{2}, A_{3}$ ) can also be used for photometric stereo estimation - as long as the four images are illuminated by a distributed light source.

\subsection{Phase Delay Estimation with Distributed Light Sources}

The classic ToF model deals with a single light source placed very close to the camera, which means (1) the extra phase delay caused by the distance between the light source and the camera is negligible, and (2) pixels in the four phase images share the same intensity value. As mentioned in Section 3.1, our approach directly uses the multiple distributed light sources for ToF sensing in order to avoid extra capturing time. Obviously, the above assumptions are violated in our case. Therefore, the classic formula used to calculate the phase delay no longer applies.

To tackle this problem, we explicitly model the phase delay caused by light positions as an unknown and use an iterative optimization scheme to solve it as well as the phase delay caused by scene depth.

Still, one can use the classic formula to generate an initial phase delay estimate, and use the light source positions to perform further iterative refinements.

The goal of each iteration is to derive the next iteration's phase delay $\phi^{\prime}$ based on the current estimate $\phi$. Given the unit camera ray $r$, and the light positions $L_{i}$, where $i=$ $\{0,1,2,3\}$ is the index of the light sources, one can write the phase delay $\alpha_{i}$ caused by the light source positions as,

$$
\left\{\begin{array}{l}
d=\frac{c \cdot \phi}{4 \pi \cdot f_{m o d}} \\
\alpha_{i}=\left(\left\|d \cdot r-L_{i}\right\|-d\right) \cdot \frac{2 \pi \cdot f_{m o d}}{c}
\end{array}\right.
$$

Assuming phase delay is caused only by light travel (in practice, one also needs to estimate an extra global phase delay caused by signal propagation inside the system). Since the ToF autocorrelation function (ACF) takes the shape of a sinusoid, one can formulate the relation between the phase delay $\phi^{\prime}$ due to depth, the phase delay $\alpha$ due to light source positions, and the ACF value $I$ as follows,

$$
A \cos \left(\frac{i \pi}{2}+\alpha_{i}+\phi^{\prime}\right)=I_{i}=\frac{I m_{\text {phase }}^{i}}{I m_{\text {intensity }}^{i}}
$$

where $A$ is the amplitude of the ACF function, $I m_{\text {phase }}$ and $I m_{\text {intensity }}$ are the intensity values in the phase and intensity images respectively. The division by $\mathrm{Im}_{\text {intensity }}^{i}$ is necessary to neutralize the differences of incident light strengths from different light sources. This equation expands to,

$$
\cos \left(\frac{i \pi}{2}+\alpha_{i}\right) \cdot A \cos \phi^{\prime}-\sin \left(\frac{i \pi}{2}+\alpha_{i}\right) \cdot A \sin \phi^{\prime}=I_{i}
$$

which in matrix form is,

$$
\left[\begin{array}{cc}
\cos \alpha_{i} & -\sin \alpha_{i} \\
\cos \left(\frac{\pi}{2}+\alpha_{i}\right) & -\sin \left(\frac{\pi}{2}+\alpha_{i}\right) \\
\cos \left(\pi+\alpha_{i}\right) & -\sin \left(\pi+\alpha_{i}\right) \\
\cos \left(\frac{3 \pi}{2}+\alpha_{i}\right) & -\sin \left(\frac{3 \pi}{2}+\alpha_{i}\right)
\end{array}\right]\left[\begin{array}{c}
A \cos \phi^{\prime} \\
A \sin \phi^{\prime}
\end{array}\right]=\left[\begin{array}{c}
I_{0} \\
I_{1} \\
I_{2} \\
I_{3}
\end{array}\right]
$$

$A \cos \phi$ and $A \sin \phi$ can be solved in the least-square sense in this overdetermined system. $\phi^{\prime}$ is then estimated as,

$$
\phi^{\prime}=\arctan \left(\frac{A \sin \phi^{\prime}}{A \cos \phi^{\prime}}\right)
$$


The refinement iterates between calculating the phase delay caused by the light source positions, i.e $\alpha_{i}$ in Eq. 4 , and the optimization for the phase delay caused by the depth via Eq. 7 and Eq. 8. The process is repeated until $\left\|\phi^{\prime}-\phi\right\|$ is smaller than a threshold, or when the number of iterations reaches a limit. Upon convergence, we will obtain a refined depth map of the scene.

\section{Fusion with Photometric Stereo}

The purpose of our novel system design is to enable fusion of position information from ToF with normal information from Photometric Stereo with only minor hardware changes. In the following, we first describe our normal map estimation via Photometric Stereo and the fusion scheme which is similar to the approach proposed by Zhang et al [10].

\subsection{Photometric Stereo with Point Light Sources}

With the assumption of directional light, the Photometric Stereo method estimates the surface normal $N$ by solving the following system based on the Lambertian assumption

$$
\left[\begin{array}{l}
V_{0}^{\top} \\
V_{1}^{\top} \\
V_{2}^{\top} \\
V_{3}^{\top}
\end{array}\right] \cdot k_{d} N=\left[\begin{array}{c}
I_{0} \\
I_{1} \\
I_{2} \\
I_{3}
\end{array}\right]
$$

where $\left\{V_{i}\right\}$ are column vectors representing the incident light directions, and $\left\{I_{i}\right\}$ are intensity values. However, with point light sources, the light direction vectors are no longer constant. Instead they need to be calculated for each pixel from its corresponding 3D coordinates $D$ and the light source positions $L$. Similar to the work by Clark [16], we further use a quadratic term to model light attenuation. Assuming the light source is infinitely small, the Lambertian shading equation can be rewritten as,

$$
V \cdot \frac{k_{d}}{\|L-D\|^{2}} N=I
$$

which in matrix form is,

$$
\left[\begin{array}{c}
V_{0, i}^{\top} \\
V_{1, i}^{\dagger} \\
V_{2, i}^{\dagger} \\
V_{3, i}^{\dagger}
\end{array}\right] \cdot k_{d} N_{i}=\left[\begin{array}{c}
I_{0} \cdot\left\|L_{0}-D_{i}\right\|^{2} \\
I_{1} \cdot\left\|L_{1}-D_{i}\right\|^{2} \\
I_{2} \cdot\left\|L_{2}-D_{i}\right\|^{2} \\
I_{3} \cdot\left\|L_{3}-D_{i}\right\|^{2}
\end{array}\right]
$$

where $V_{k, i}=L_{k}-D_{i}$ and $D_{i}$ represents the $3 \mathrm{D}$ coordinates estimated by the ToF principle described in Section 3.3. Now, the surface normal can be estimated efficiently by solving this linear system.

\subsection{Fusion of depth and normal}

The position information from range map and the surface normal from Photometric Stereo is combined using a framework similar to [10]. In their approach, they iterative refine the position and the normal. During the position refinement step, the following objective function is minimized

$$
\begin{aligned}
E= & \sum_{i j} \mu_{i j}\left(D_{i j}-D_{i j}^{0}\right)^{2}+\lambda_{p} \sum_{i j} \mu_{i j}\left(\left(N_{i j}^{0} \cdot \frac{\partial D}{\partial u}\right)^{2}+\right. \\
& \left.\left(N_{i j}^{0} \cdot \frac{\partial D}{\partial v}\right)^{2}\right)+\lambda_{s} \nabla D
\end{aligned}
$$

where $D_{i j}^{0}$ and $N_{i j}^{0}$ are the original depth and normal, which are obtained via ToF sensing in Section 3.3 and Photometric Stereo in Section 4.1. $\left\{\mu_{i j}\right\}$ are per-pixel weights and $\frac{\partial D}{\partial u}$ and $\frac{\partial D}{\partial v}$ are derivatives of the depth map along the image grid. The last term ensures smoothness via the Laplacian operator $\nabla . \lambda_{p}$ and $\lambda_{s}$ leverage the relative importance across different terms. After this step, the normal is updated via interpolation between $N^{0}$ and the one calculated from the refined depth map. Furthermore, a Gaussian filter is used in the differential operator.

To better suppress the noise in the raw depth produced by $\mathrm{ToF}$ and reveal more details preserved in the normal map, we first smooth the depth data with Bilateral Filtering. Moreover, instead of using a global weight between the first term and the second term, we use a per-pixel adaptive weighting scheme to take into considerations the flatness of the surface. That is, the weight $\lambda_{p}$ is adapted for each pixel. The idea is to better preserve the details already exist in the depth map and then use the normal map to refine the regions with less details. Therefore, the higher the curvature, the more weight is put on depth, and when the surface is more flat, more weight is assigned to normal. In essence, the weight for depth is the second derivative of the plane pixels plus a small base value, to prevent the weight to be zero. This iterative optimization process is performed until no further improvement on the depth map is gained.

\section{Light source calibration}

The entire procedure described so far requires knowledge of the light source positions. The usual method of calibration using chrome spheres is unsuitable in this situation, since the only sources of illumination are the IR LEDs connected with the ToF camera, and the reflectiveness of the sphere makes its position hard to detect, as shown in Figure 3. Also, the lower resolution of the sensor favors planar objects to spherical ones.

In this section, we describe two proposed light position calibration methods for users to choose from based on the availability of the required auxiliary objects. Both of them take advantage of the point light property. In both methods, checker patterns cover part of the planar objects to help estimate their poses. The plane parameters are $(z, \theta, \phi)$, where $z$ indicates depth from the camera center, and the normal of the plane is $(\cos \theta \sin \phi, \sin \theta \sin \phi, \cos \phi)$. These parame- 


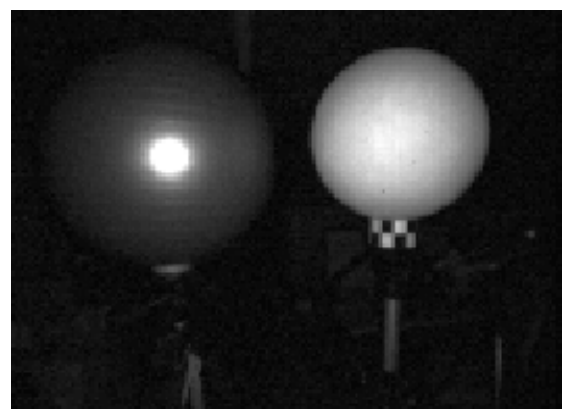

Figure 3. Image of a semi-reflective sphere next to a diffuse one. They have roughly the same distance to the camera.

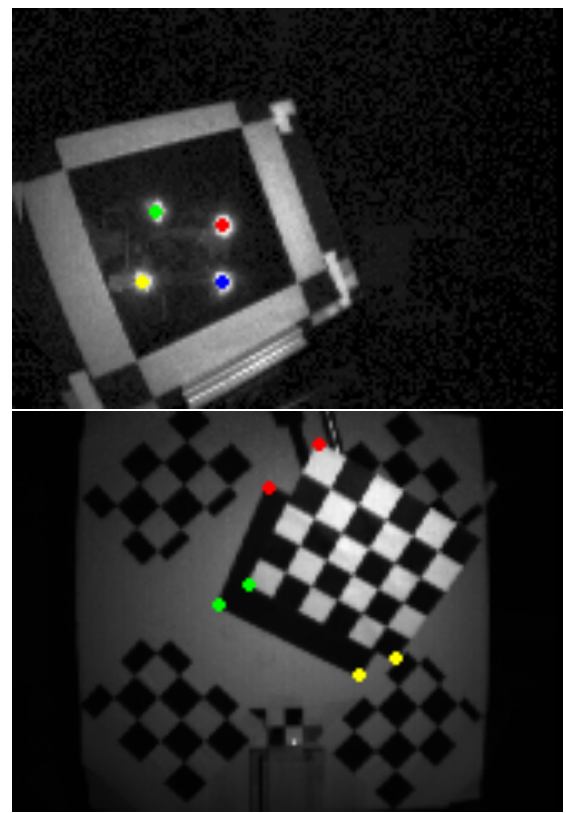

Figure 4. Illustration of our two calibration methods, (top, showing 4 lights) using a flat mirror and (bottom, showing one light) via shadow casting. Interest points for triangulating the light positions are marked in color.

ters are estimated by minimizing the following cost function,

$$
\min \sum_{i, j}\left(D\left(P\left(r_{i}, z, \theta, \phi\right), P\left(r_{j}, z, \theta, \phi\right)\right)-d_{i j}\right)^{2}
$$

where $D\left(P_{1}, P_{2}\right)$ is the Euclidean distance between two 3D points, $P(r, z, \theta, \phi)$ is the intersection point of the camera ray $r$ and the plane $(z, \theta, \phi)$, and $d_{i j}$ is the measured distance between the $i$-th and $j$-th feature point on the plane.

\subsection{Calibration with flat mirror}

The idea of the first method is to triangulate the light position by tracing reverse light rays emitted from the camera and the one reflected by the mirror. The light source forms a single bright dot when inside the view of the mirror. With multiple mirror poses, the rays reflected from those points

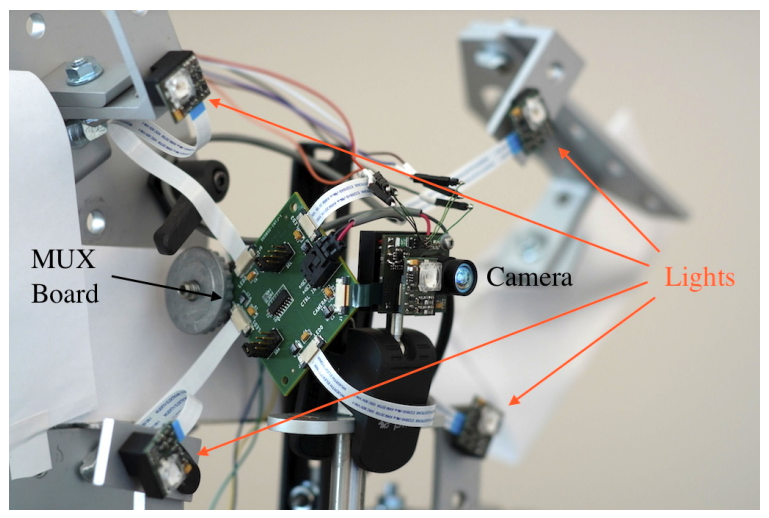

Figure 5. Our novel capturing setup that consists of a ToF sensor and four point light sources.

are intersected to find the position of the light, as shown in Fig. 4(top). The reflection point $(x, y)$ in the image is calculated by taking a weighted mean of the brightest connected pixels in the mirror area,

$$
(x, y)=\sum_{i}\left(x_{i}, y_{i}\right) \cdot I\left(x_{i}, y_{i}\right)
$$

where $I(x, y)$ is the intensity at $(x, y)$ and serves as the confidence. This method is simple, only requiring a flat mirror, and easy to operate.

\subsection{Calibration via Shadow Casting}

Our second method uses two opaque planar surfaces. A relatively small, thin, rectangular foreground plane is placed in between the camera/light setup and a larger background screen, as shown in Fig. 4(bottom). When the light is activated, the foreground plane casts shadows on the background plane. The corners of the shadow could be located using any corner detector. Corners on the foreground plane could be found either by corner detectors, or extrapolated from the checker pattern, if the edges of the plane coincide with edges of the pattern. The foreground plane may be intelligently positioned so that more than one corner/shadow pair are reliably detected in a single image, which enables the light position to be determined in one shot. As this method does not require a reflective object, it can avoid inaccuracies induced by imperfect mirror surfaces. Experimentally, we found that this method achieves slightly higher accuracy compared to the first one. However, this require opaque planar surfaces and the foreground plane to have sharp corners for clear shadow casting.

\section{Experiments}

\subsection{Time-of-Flight results}

This section examines the performance of the ToF algorithm for distributed light sources against the original $\mathrm{ToF}$ 


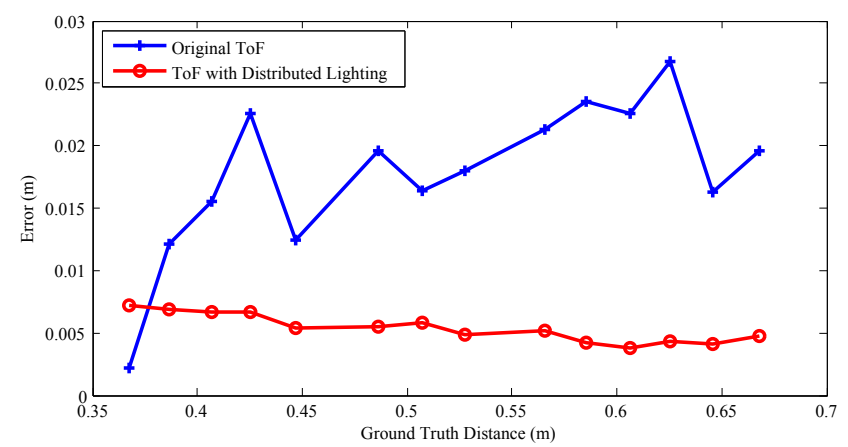

Figure 6. Comparison of accuracy in distance measurement with original ToF v.s. distributed lighting.

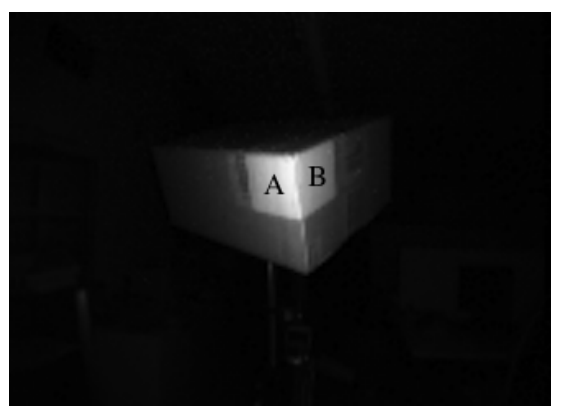

Figure 7. Two orthogonal planes A and B, for measuring angular error.

mechanism. We modified a PMD sensor which has a detachable lighting module. The lighting module is connected with the main board via s synchronization cable. We built a 1-to-4 demultiplexer (DEMUX) board so that the four LEDs are turned on sequentially. With this modified ToF sensor we can perform our experiments.

Our first experiments is to measure the distance accuracy. Firstly, we use the new setup to capture phase and intensity images of a flat plane over a range of distances where its position can be accurately determined by precision measurement. The ground truth plane parameters are obtained by fitting checker patterns on it. The depth data in both algorithms are smoothed with bilateral filter, in order to exclude ToF noise from the inaccuracy. For both setups, pixels from the center area of the plane are compared with the ground truth plane, and the average distance error is plotted in Figure 6.

In our second experiments, we discover the angular accuracy of the ToF algorithm for distributed light sources. Two sides of a box resembling two approximately orthogonal planes are fitted both in 2D (as ground truth) and depth values from both setups, as illustrated in Figure 7 . The results are listed in Table 1 . They show that the angular error by the new setup is comparable to the original $\mathrm{ToF}$ camera.
Table 1. Comparison of angular error with original ToF v.s. distributed lighting.

\begin{tabular}{lccc}
\hline$\angle A B$ & Ground Truth & Sensor Result & Error \\
\hline Original ToF & $89.07^{\circ}$ & $96.75^{\circ}$ & $7.68^{\circ}$ \\
Distd. Lights & $89.45^{\circ}$ & $97.57^{\circ}$ & $8.12^{\circ}$ \\
\hline
\end{tabular}
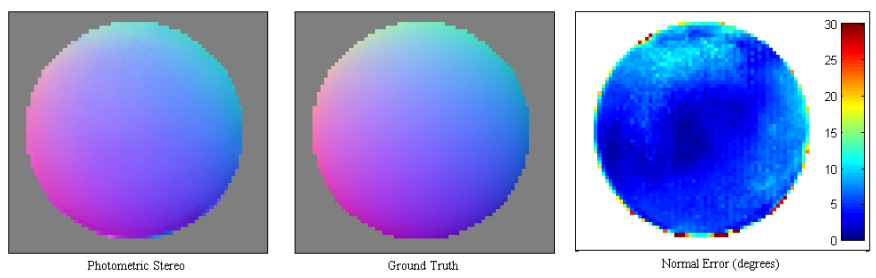

Figure 8. Analysis of normal accuracy. From left to right are normal field by PS, ground truth generated from the sphere's position, and error map of the PS result.

\subsection{Photometric stereo results}

In this section, we examine the accuracy of the normal field generated by photometric stereo using a sphere. The sphere is located by its contour in a stereo view and is taken phase and intensity images. The normal map calculated by our photometric stereo method based on depth, in comparison with the ground truth normal map, is shown in Figure 8. Errors in the bottom row of pixels are caused by the supporting stand immediately below the sphere.

\subsection{Fusion results}

We perform the fusion algorithm on a number of objects, and results from various stages of the pipeline are displayed here. It can be noticed that the mesh generated from the distributed light ToF setup looks somewhat more noisy than the original ToF depth map, as there are hardware optimizations in the ToF camera that are not readily known and therefore not replicated in our algorithm. However, as the depth map is smoothed before fusing with the normal map, we can still obtain a high level of detail, and generate true-to-life fusion meshes. Holes in the depth maps from the distributed lights setup are due to 1) long faces in the mesh are removed by the fusion algorithm, and 2) non-Lambertian parts of the surface (such as the eyes in the human head model) causes wrong depth and normal. Finally, our PMD Camboard nano ToF camera requires firmware refreshing between depth and intensity captures, therefore it is not currently possible to capture dynamic footage.

\section{Conclusion}

In this paper, we present a novel use of a phase-shiftbased time-of-flight sensor. We notice that such a sensor requires four different phase images to calculate a depth 

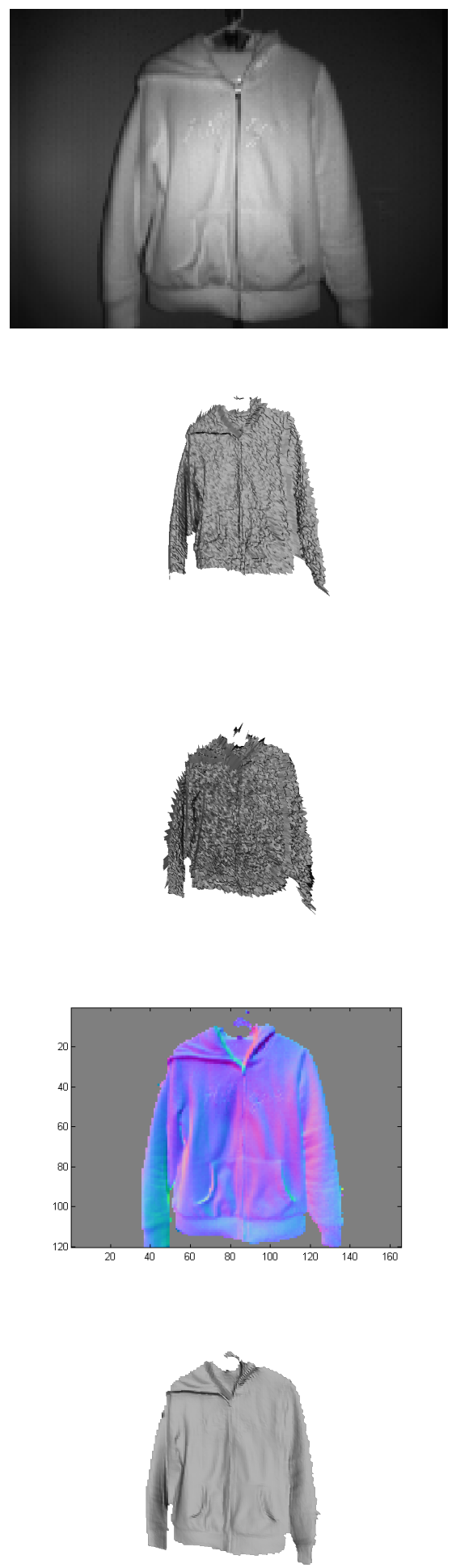

Figure 9. Results of a hanging coat. From top down: intensity image, mesh from original ToF, mesh from ToF with distributed lighting, normal map, final mesh from fusion with PS.
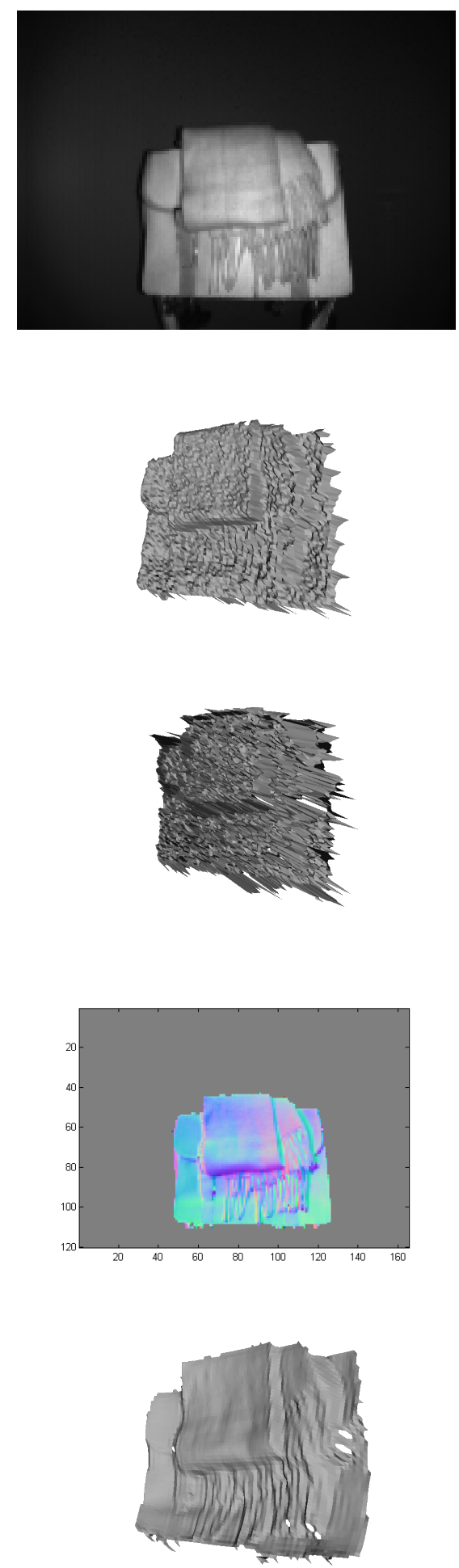

Figure 10. Results of a scarf draped over a bag. From top down: intensity image, mesh from original ToF, mesh from ToF with distributed lighting, normal map, final mesh from fusion with PS. 

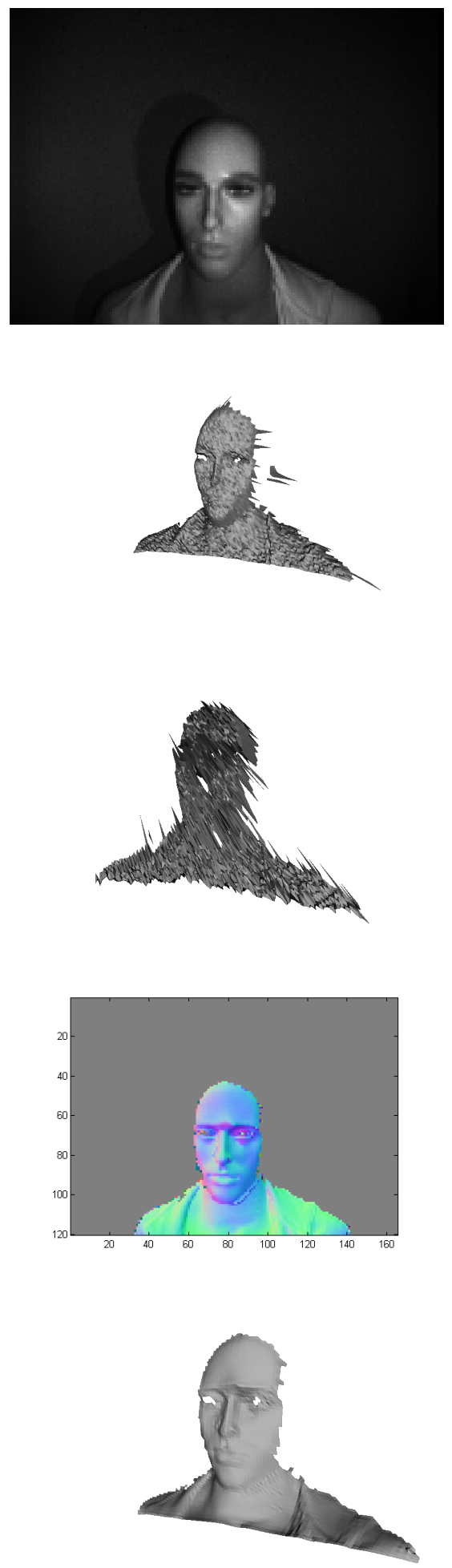

Figure 11. Results of a human head model. From top down: intensity image, mesh from original ToF, mesh from ToF with distributed lighting, normal map, final mesh from fusion with PS. value. Photometric stereo also requires three or more images. The only difference is that photometric stereo requires different light source locations while a ToF system typically keep the light fixed for the ease of calculation. We have developed a time-of-flight algorithm that can utilize lights from different locations. Therefore by simply adding three more lights and a switch board to alternate the light source, we can use the same sensor to capture both depth and surface normal at the same time. Applying sensor fusion on the output leads to much improved depth map with metric accuracy and small surface details. We demonstrate the capability of our novel hardware/software system with a number of quantitative and qualitative experiments. Looking into the future we hope to explore a multi-light multiToF-sensor setup to further improve the quality of the depth map and allow the system to scale up.

\section{Acknowledgements}

This work is supported in part by US National Science Foundation grants IIS-123154 and IIS-1208420 and Natural Science Foundation of China grant 61332017. Ruigang Yang is the corresponding author for this paper.

\section{References}

[1] Xu, Z., Schwarte, R., Heinol, H., Buxbaum, B., Ringbeck, T.: Smart pixel - photonic mixer device (PMD). In: Proc. Int. Conf. on Mechatron. \& Machine Vision. (1998) 259-264

[2] Oggier, T., Büttgen, B., Lustenberger, F., Becker, G., Rüegg, B., Hodac, A.: Swissranger sr3000 and first experiences based on miniaturized 3D-ToF cameras. In: Proc. of the First Range Imaging Research Day at ETH Zurich. (2005)

[3] Cui, Y., Schuon, S., Chan, D., Thrun, S., Theobalt, C.: 3d shape scanning with a time-of-flight camera. In: Computer Vision and Pattern Recognition (CVPR), 2010 IEEE Conference on, IEEE (2010) 1173-1180

[4] Van Ouwerkerk, J.: Image super-resolution survey. Image and Vision Computing 24(10) (2006) 10391052

[5] Schuon, S., Theobalt, C., Davis, J., Thrun, S.: Lidarboost: Depth superresolution for tof 3d shape scanning. In: Computer Vision and Pattern Recognition, 2009. CVPR 2009. IEEE Conference on, IEEE (2009) $343-350$

[6] Yang, Q., Yang, R., Davis, J., Nistér, D.: Spatial-depth super resolution for range images. In: Computer Vision and Pattern Recognition, 2007. CVPR'07. IEEE Conference on, IEEE (2007) 1-8 
[7] Kopf, J., Cohen, M.F., Lischinski, D., Uyttendaele, M.: Joint bilateral upsampling. In: ACM Transactions on Graphics (TOG). Volume 26., ACM (2007) 96

[8] Kornprobst, P., Tumblin, J.: Bilateral filtering: Theory and applications. Now Publishers Inc (2009)

[9] Nehab, D., Rusinkiewicz, S., Davis, J., Ramamoorthi, R.: Efficiently combining positions and normals for precise 3d geometry. In: ACM Transactions on Graphics (TOG). Volume 24., ACM (2005) 536-543

[10] Zhang, Q., Ye, M., Yang, R., Matsushita, Y., Wilburn, B., Yu, H.: Edge-preserving photometric stereo via depth fusion. In: Computer Vision and Pattern Recognition (CVPR), 2012 IEEE Conference on, IEEE (2012) 2472-2479

[11] Godbaz, J.P., Cree, M.J., Dorrington, A.A.: Closedform inverses for the mixed pixel/multipath interference problem in amcw lidar. Volume 8296. (2012) 829618-829618-15

[12] Dorrington, A.A., Godbaz, J.P., Cree, M.J., Payne, A.D., Streeter, L.V.: Separating true range measurements from multi-path and scattering interference in commercial range cameras. Volume 7864. (2011) 786404-786404-10

[13] Bhandari, A., Kadambi, A., Whyte, R., Barsi, C., Feigin, M., Dorrington, A.A., Raskar, R.: Resolving multi-path interference in time-of-flight imaging via modulation frequency diversity and sparse regularization. CoRR abs/1404.1116 (2014)

[14] Heide, F., Xiao, L., Kolb, A., Hullin, M.B., Heidrich, W.: Imaging in scattering media using correlation image sensors and sparse convolutional coding. Opt. Express 22(21) (Oct 2014) 26338-26350

[15] Heide, F., Hullin, M.B., Gregson, J., Heidrich, W.: Low-budget transient imaging using photonic mixer devices. ACM Trans. Graph. 32(4) (July 2013)

[16] Clark, J.J.: Active photometric stereo. In: Computer Vision and Pattern Recognition, 1992. Proceedings CVPR'92., 1992 IEEE Computer Society Conference on, IEEE (1992) 29-34 